\title{
THE $K$ THEORY OF THE PROJECTIVE UNITARY GROUPS
}

BY T. PETRIE

Communicated by E. Lyle, May 9, 1966

Let $U=U\left(p^{r}\right)$ be the unitary group on complex $p^{r}$ space, $p$ an odd prime. Let $S^{1} \subset U$ be the set of matrices $\lambda I$ where $\lambda$ is a complex number with $|\lambda|=1$ and $I$ is the identity matrix. Then $S^{1}$ is the center of $U$ and $P U\left(p^{r}\right)=P U=U / S^{1}$.

We determine the complex $K^{*}$ groups for the spaces $P U$ by first determining the $\bmod q K^{*}$ groups of these spaces [2] then using the $\bmod p$ Bockstein spectral sequence to obtain the $p$ torsion. $K^{*}\left[P U\left(p^{r}\right)\right]$ and $H^{*}\left[P U\left(p^{r}\right)\right]$ have no $q$ torsion for $q \neq p$ and the $\bmod p$ Bockstein spectral sequences for these two groups are isomorphic; thus,

Theorem 5.5. $H^{*}\left[P U\left(p^{r}\right), Z\right]$ and $K^{*}\left[P U\left(p^{r}\right)\right]$ are isomorphic as abelian groups.

The details of these proofs will be published elsewhere. The outline follows:

Let $B_{S^{1}}$ and $B_{U}=B_{U}\left(p^{r}\right)$ be the classifying spaces of the indicated groups. There are the following maps

$$
U \stackrel{f}{\rightarrow} P U \stackrel{i}{\rightarrow} B_{S^{1}} \stackrel{B_{\Delta}}{\longrightarrow} B_{U} .
$$

Either $i$ or $B_{\Delta}$ may be considered fibrations. We use the following diagram

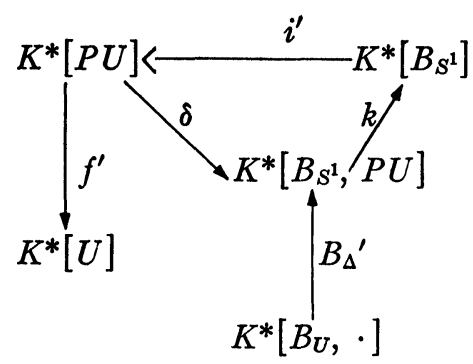

Let $\rho_{q}: K^{*}[, Z] \rightarrow K^{*}\left[, Z_{q}\right]$ be the reduction [2] and $\beta_{K}: K\left[, Z_{q}\right]$ $\rightarrow K[, Z]$ the Bockstein. $\exists \sigma_{1}, \sigma_{2}, \cdots, \sigma_{p^{r}} \in K^{*}\left[B_{U}, \cdot\right] \ni K^{*}\left[B_{U}\right]$ $=Z\left[\left[\sigma_{1}, \cdots, \sigma_{p^{r}}\right]\right], H^{*}\left[B_{U}\right]=Z\left[\bar{\sigma}_{1}, \cdots, \bar{\sigma}_{p^{r}}\right], K^{*}[U]=E\left[s \sigma_{1}, \cdots, s \sigma_{p^{r}}\right]$, $K^{*}\left[B_{S^{1}}\right]=Z[[y]], H^{*}\left[B_{S^{1}}\right]=Z[\bar{y}] . \rho_{p}$ is onto for these groups and it will be convenient to use $x$ for $\rho_{p}(x)$ when possible. $k B_{\Delta}^{\prime}\left(\sigma_{i}\right)=C_{p^{r}, i} y^{i}$ and $B_{\Delta}^{*}\left(\bar{\sigma}_{\imath}\right)=C_{p^{r}, i} \bar{y}^{i}$. 
Since $k B_{\Delta}^{\prime}\left(\sigma_{i}\right)=C_{p^{r}, i} \bar{y}^{i}, k B_{\Delta}^{\prime}\left(\sigma_{i}\right)=0 \bmod p$ for $i<p^{r}$; hence it follows from (1) using $Z_{p}$ coefficients that for $i<p^{r} \exists x_{i} \in K^{*}\left[P U, Z_{p}\right]$ such that $\delta x_{i}=B_{\Delta}^{\prime} \rho_{p}\left(\sigma_{i}\right)$. Let $J$ be the set of integers $j$ such that $1<j<p^{r}$ and $j$ is not a $p$ th power. A set $Y=\left\{Y_{j} \mid j \in J\right\} \subset K^{*}\left[P U, Z_{p}\right]$ is defined. Let $X=\left\{x_{p^{i}} \mid i=0,1, \cdots, r-1\right\}, w=i^{\prime}(y)$ and $\Lambda=E[Y] \otimes Z_{p}[w] / w^{p^{r}}$.

TheOREM 2.6. $K\left[P U, Z_{p}\right]=E[X] \otimes \Lambda$ as an algebra. $\Lambda$ is in the image of $\rho_{p}$ so that $\beta_{K}(\Lambda)=0$.

Proof. The $E_{2}$ term of the $\bmod p$ spectral sequence arising from the fibration $U \rightarrow P U \rightarrow B_{S^{1}}$ is $E_{2}=E\left[s \sigma_{1}, \cdots, s \sigma_{p^{r}}\right] \otimes Z_{p}[\bar{y}]$. Since $\delta x_{i}=B_{\Delta}^{\prime} \rho_{p}\left(\sigma_{i}\right)$ it readily follows that $f^{\prime}\left(x_{i}\right)=s \sigma_{i}$ for $i<p^{r} \therefore d_{j}\left(s \sigma_{i}\right)=0$ for all $j$ and $i<p^{r}$. Since $B_{\Delta}^{*}\left(\bar{\sigma}_{p^{r}}\right)=\bar{y}^{p^{r}}$ it follows that $d_{2 p^{r}}\left(s \sigma_{p^{r}}\right)=\bar{y}^{p^{r}}$. This describes the spectral sequence and we find that there is a filtration of $K^{*}\left[P U, Z_{p}\right]$ whose associated graded module $E_{0} K^{*}\left[P U, Z_{p}\right]$ is $E\left[s \sigma_{1}, \cdots, s \sigma_{p^{r-1}}\right] \otimes Z_{p}[\bar{y}] / \bar{y}^{p^{r}}$.

It is easy to see that the $x_{p^{i}} \in K^{*}\left[P U, Z_{p}\right]$ represent $s \sigma_{p^{i}}$ in $E_{0} K^{*}\left[P U, Z_{p}\right]$. The $y_{j}$ where chosen to represent the $s \sigma_{j}$ for $j \in J$. $w$ represents $\bar{y}$. This information is sufficient to show that the obvious map of $E[X] \otimes \Lambda$ to $K^{*}\left[P U, Z_{p}\right]$ is an isomorphism of algebras.

The appropriate tool for relating the Bockstein $\beta_{H}$ in ordinary cohomology theory to the Bockstein $\beta_{K}$ in $K$ theory is the AtiyahHirzebruch spectral sequence. It is convenient to use the approach [10] for obtaining this spectral sequence.

Let $u$ be the infinite unitary group. Spaces $B_{\mathcal{U}}(2 i+2)$ are inductively defined by killing the $2 i$ th homotopy group of $B_{\mathcal{U}}(2 i)$ for $i \geqq 1$ and $B_{\mathcal{U}}(2)=B_{\mathfrak{u}}$. In particular there is a commutative diagram of fiber spaces

$$
\begin{aligned}
& K[Z, 2 i] s^{1} \stackrel{h_{i}}{\longrightarrow} B \mathfrak{u}(2 i+2) \stackrel{f_{i+1}}{\longrightarrow} B \mathfrak{u}(2 i) \\
& \begin{array}{ccc}
\downarrow & \downarrow \psi_{i+1} & \downarrow g_{i} \\
K[Z, 2 i]^{S^{1}} \longrightarrow & P K[Z, 2 i] & \longrightarrow K[Z, 2 i]
\end{array}
\end{aligned}
$$

where $K[Z, 2 i]$ is the indicated Eilenberg MacLane space with path space $P K[Z, 2 i]$. Define $D^{2 j}{ }^{q}[X, Z]=\left[X, B_{\mathfrak{u}}(2 j)^{S^{q}}\right] j \geqq 1, q=0,1$. $E_{1}^{2 j, q}[X, Z]=\left[X, K[Z, 2 j]^{s^{q}}\right] j \geqq 1, q=0,1 . D=\sum D^{2 j, q}, E_{1}=\sum E_{1}^{2 j, q}$. The maps in diagram (2) allow us to define an exact couple

$$
\begin{gathered}
D[X, Z] \stackrel{i}{\longrightarrow} D[X, Z] \\
\nwarrow k \\
E_{1}[X, Z]=\tilde{H}[X, Z] .
\end{gathered}
$$


Define

$$
\begin{array}{ll}
D^{2 j, q}\left[X, Z_{p}\right]=\left[X, B u(2 j+2)^{Z(p) \wedge S^{q}}\right] & i \geqq 0, q=0,1, \\
E_{1}^{2 j, q}\left[X, Z_{p}\right]=\left[X, K[Z, 2 i+2]^{Z(p) \wedge S^{q}}\right] & i \geqq 0, q=0,1
\end{array}
$$

where $Z(p)=E^{2} U_{p} S^{1}$. As above we obtain an exact couple

$$
\begin{array}{cc}
D\left[X, Z_{p}\right] \stackrel{i_{p}}{\longrightarrow} D\left[X, Z_{p}\right] \\
\nwarrow k_{p} & j_{p} \swarrow \\
E_{1}\left[X, Z_{p}\right]=\widetilde{H}\left[X, Z_{p}\right] .
\end{array}
$$

Let $E_{r}[X, Z]$ be the spectral sequence associated with the couple (3) and $E_{r}\left[X, Z_{p}\right]$ the spectral sequence associated with the couple (4). The first converges to $\tilde{K}^{*}[X, Z]$ and the second to $\tilde{K}^{*}\left[X, Z_{p}\right]$.

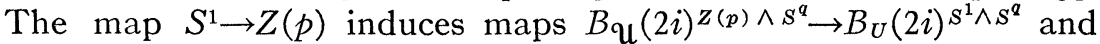
$K[Z, 2 i] Z(p) \wedge S^{1} \rightarrow K[Z, 2 i]^{1}+S^{q}$ which in turn define a map of the exact couple (4) into the exact couple (3). $\beta_{r}: E_{r}\left[X, Z_{p}\right] \rightarrow E_{r}[X, Z]$ is the induced map of spectral sequences. We show that $\beta_{1}=\beta_{H}$ and that $\beta_{\infty}=E_{0}\left(\beta_{K}\right): E_{0} \widetilde{K}^{*}\left[X, Z_{p}\right] \rightarrow E_{0} \widetilde{K}^{*}[X, Z]$.

Theorem 2.6 implies that the spectral sequence $E_{r}\left[P U, Z_{p}\right]$ $\Rightarrow \tilde{K}\left[P U, Z_{p}\right]$ collapses so that $H\left[P U, Z_{p}\right]=E_{\infty}\left[P U, Z_{p}\right]$ $=E_{0} K^{*}\left[P U, Z_{p}\right]$ and $\beta_{H}=\beta_{\infty}$. Let $\bar{x}_{p^{i}}, \bar{w} \in H^{*}\left[P U, Z_{p}\right]$ be the elements represented in $E_{0} K^{*}\left[P U, Z_{p}\right]$ by $x_{p^{i}}, w \in K^{*}\left[P U, Z_{p}\right]$. Then $\beta_{H}\left(\bar{x}_{p^{i}}\right)=\lambda_{i, 0} z \bar{v}^{p^{i}}$ and the highest power of $p$ dividing $\lambda_{i, 0}$ is $p^{r-i-1}$. One observes that $\delta \beta_{K}\left(x_{p^{i}}\right)=0$ so that $\beta_{K}\left(x_{p^{i}}\right) \in \operatorname{Im} i^{\prime}$; thus $\beta_{K}\left(x_{p^{i}}\right)=\alpha_{i} w^{p^{i}}$ $+\sum_{j>0} \lambda_{i, j} w^{p^{i}+j}$. It follows that $E_{0}\left(\beta_{K}\right) \bar{x}_{p^{i}}=\alpha_{i} \bar{w}^{p^{i}}$ in $E_{0} K^{*}[P U, Z]$. $E_{0}\left(\beta_{K}\right)=\beta_{\infty}=\beta_{H}$ implies that $\lambda_{i, 0}=\alpha_{i}$.

We must find a new set of generators $z_{p^{i}}$ of the algebra $K^{*}\left[P U, Z_{p}\right]$ to replace the $x_{p^{i}}$ and so that $\beta_{K}\left(z_{p^{i}}\right)=\lambda_{i, 0} w^{p^{i}}$. Precisely

THEOREM 4.4. There is a subset $Z_{0}=\left\{z_{p^{i}} \mid i=0,1, \cdots, r-1\right\}$ of $K^{*}\left[P U, Z_{p}\right]$ such that $K^{*}\left[P U, Z_{p}\right]$ is isomorphic to $E\left[Z_{0}\right] \otimes \Lambda$ as an algebra and such that $\beta_{K}(\Lambda)=0, \beta_{K}\left(Z_{p^{i}}\right)=\lambda_{\imath, 0} w^{p^{i}}$.

Theorem 4.4 gives sufficient information to determine the $\bmod p$ Bockstein spectral sequence of $K^{*}[P U, Z]$. Let $U_{k}$ be the set of

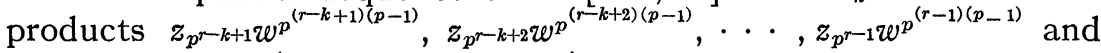
$W_{k} \subset Z_{0}$ the set $\left\{z_{1}, z_{p}, \cdots, z_{p^{r-k-1}}\right\}$. In particular, $U_{1}=0, U_{r+1}=U_{\infty}$ and $W_{r}=0$.

THEOREM 4.5. The kth term of the Bockstein spectral sequence for $K^{*}[P U, Z]$ is

(i) $E_{k}=E[Y] \otimes E\left[W_{k}\right] \otimes E\left[U_{k}\right] \otimes\left\{E\left[z_{p^{r-k}}\right] \otimes Z_{p}[w] / w^{p^{r-k+1}}\right\}$ 
(ii) $\beta_{k}$, the kth differential, is a derivation, $\beta_{k}=0$ in $E[Y] \otimes E\left[W_{k}\right]$ $\otimes E\left[U_{k}\right]$ and $\beta_{k}\left(z_{p^{r-k}}\right)=\lambda_{r-k, 0}^{1} w^{p^{r-\alpha}}$ for $\lambda_{r-k, 0}^{1}=\left(1 / p^{k-1}\right) \lambda_{r-k, 0}$.

Theorems 4.4 and 4.5 hold for the ordinary cohomology of $P U$. In particular, the $\bmod p$ Bockstein spectral sequences for $K^{*}[P U, Z]$ and $H^{*}[P U, Z]$ are isomorphic.

\section{REFERENCES}

1. D. Anderson, $\mathrm{Ph} . \mathrm{D}$. Thesis, Harvard University, Cambridge, Mass., 1962.

2. S. Araki and $\mathrm{H}$. Toda, Multiplicative structures in Mod $q$ cohomology theories. I, Osaka Math. J. 2 (1965), 71-115.

3. M. Atiyah and F. Hirzebruch, Vector bundles and homogeneous spaces, Proc. Sympos. Pure Math., Vol. 3, Amer. Math. Soc., Providence, R. I., 1961.

4. M. Atiyah, On the K theory of compact Lie groups, Topology 4 (1965), 95-99.

5. P. Baum and W. Browder, The cohomology of quotients of classical groups, Topology 3 (1965), 305-336.

6. P. Baum, Cohomology of homogeneous spaces, $\mathrm{Ph} . \mathrm{D}$. Thesis, Princeton University, Princeton, N. J., 1963.

7. A. Borel, Sur la cohomologie des espaces fibres principaux et des espaces homogènes de groupes de Lie compact, Ann. of Math. 57 (1953), 115-207.

8. A. Dold, Half exact functors and cohomology, Summer Topology Institute, Seattle, 1963 (mimeographed notes of the Amer. Math. Soc.) $1-23$.

9. S. Eilenberg and J. Moore, Limits and spectral sequences, Topology 1 (1961),

10. E. Thomas, $A$ spectral sequence for $K$-theory, Appendix II, Lectures on $K[X]$ by R. Bott, Harvard University.

Institute for Defense Analyses 